\title{
具有激动过氧化物酶体增殖物激活受体活性的5- 芳(杂环)亚甲基噻唑烷-2,4-二酮类化合物的研究
}

\author{
黄敏 ${ }^{\dagger}, 弓$ 坤 $^{\dagger}$, 范莉, 周礼江, 杨大成 ${ }^{*}$ \\ 生物有机与药物化学研究所; 重庆市高校应用化学实验室; 西南大学化学化工学院, 重庆 400715 \\ †同等贡献 \\ *通讯作者, E-mail: hxydc@swu.edu.cn
}

收稿日期: 2017-04-09; 接受日期: 2017-04-25; 网络版发表日期: 2017-07-21

国家自然科学基金(编号: 21542003)资助项目

\begin{abstract}
摘要根据药物设计基本原理, 以噻唑烷-2,4-二酮为基本结构单元设计合成了目标分子 TM1 TM3 系列. 通过体外过氧化物酶体增殖物激活受体反应元件(PPRE)活性测定, 篮选出 4 个PPRE相对激动活性分别为 $105.76 \% 、 118.15 \%$ 、 $125.3 \%$ 和 $100.53 \%$ 的分子IM2、IM3、TM2c和 TM3c (参考药物吡格列酮, 100\%); 进一 步, 设计合成了 IM2 与IM3 的衍生物TM4 与 TM5 系列, 发现了 16 个PPRE相对激动活性高于 $100 \%$ 的 TM4 系列 分子, 其中 TM4i和 TM4y的PPRE活性最高, 分别为 $239.77 \%$ 和 $204.70 \%$. 毒性预测显示, 高活性分子毒性较小. 本研究发现了潜在的过氧化物酶体增殖物激活受体(PPAR)先导分子, 为糖尿病药物的研究提供了新的思路.
\end{abstract}

关键词糖尿病, 过氧化物酶体增殖物激活受体, 兴莝烷-2,4-二䣳, 毒性预测, 合成

\section{1 引言}

糖尿病(diabetes mellitus, DM) 是一种全身性慢性 代谢紊乱疾病, 我国非胰岛素依赖型糖尿病(T2DM) 患者占总患病人数的 $90 \%$ 以上 ${ }^{[1]}$. 胰岛素增敏剂是使 胰岛素发挥正常作用的一类化学物质, 它可改善胰岛 素的敏感状态. 胰岛素增敏剂主要有噻唑烷二酮类 (TZDs)和双胍类. 1997年1月, 美国食品药品监督管理 局(Food and Drug Administration, FDA)批准曲格列酮 (troglitazone)在日本、美国和英国上市销售 ${ }^{[2]}$, 是世界 上第一个临床使用的TZDs胰岛素增敏剂. 但随后发现 该药肝毒性大 ${ }^{[3]}$ 并有临床死亡病例的报告, 因此于 2000 年停止使用. 1999年, 美国FDA先后批准胰岛素增敏 剂罗格列酮(rosiglitazone) $)^{[4]}$ 与吡格列酮(pioglitazone) ${ }^{[5]}$ 上市销售. 虽然二者显示良好的降糖效果, 但长期、 大剂量服用的患者普遍存在体重增加、浮肿、低密 度脂蛋白 (LDL)胆固醇水平升高 ${ }^{[6]}$ 等副作用. 此外, 现 有TZDs药物还存在合成工艺较为繁琐、部分反应条 件较为苛刻、对合成设备要求较高等缺点. 因此, 设 计和开发高效低毒、合成简易、成本低廉的TZDs药 物, 成为解决TZDs药物不足的重要任务.

目前, 上市的TZDs药物分子的结构由 3 部分组成 (图1): 亲水性 TZD结构 $\mathrm{A}$, linker结构 $\mathrm{B}$, 疏水性片段 C. 本实验室曾设计合成了含 5-芳(杂环)亚甲基噻唑烷2,4-二酮结构单元的化合物, 发现了体外 $\alpha$-葡萄糖苷 酶抑制活性、过氧化物酶体增殖物激活受体反应元

引用格式: 黄敏, 张坤, 范莉, 周礼江, 杨大成. 具有激动过氧化物酶体增殖物激活受体活性的5-芳(杂环)亚甲基噻唑烷-2,4-二酮类化合物的研究. 中国科学: 化学, 2017, 47: 1114-1122

Huang M, Zhang K, Fan L, Zhou L, Yang D. Design, synthesis and PPAR activities of 5-arylmethylene and 5-heteroaromatic methylene thiazolidine-2,4-diones. Sci Sin Chim, 2017, 47: 1114-1122, doi: 10.1360/N032017-00070 
件(PPRE)激动活性和蛋白质酪氨酸磷酸酶1B抑制活 性较好的化合物 ${ }^{[7,8]}$; 构效关系表明, $\mathrm{A}$ 部分的氨基修饰 后, 化合物的PPRE激动活性降低. 基于此, 我们采用 保留 $\mathrm{A}$ 部分 $\mathrm{TZD}$ 单元、 $\mathrm{C}$ 部分为与上市药物最为接近 的苯基结构、 $\mathrm{B}$ 部分左端为 $\mathrm{CH}_{2} \mathrm{O}$ (缩短linker) 且右端 为刚性 $\mathrm{CH}=$ 结构单元的分子设计策略, 尝试性地设计 了目标化合物 TM1系列(图1 $)^{[9 \sim 11]}$.

体外活性数据显示, 目标化合物 TM1系列的PPRE 相对激动活性较差(最高为 $64.99 \%$ ), 随后尝试将 $\mathrm{C}$ 部 分改为长度不同的烷基, 设计了目标化合物 TM2 系列 (图2). 活性数据显示, 当烷基链长为 2 时, 分子的PPRE 相对激动活性达到 $125.3 \%$. 循此思路, 为了进一步降 低分子的 $C \log P$, 我们在 $\mathrm{C}$ 部分烷基链上引入羟基, 通 过增加亲水性以获得活性更高的分子, 即目标化合物 TM3系列(图2).

在活性测试时意外发现, 中间体 IM2与IM3 (图3) 的活性分别达到 $105.76 \%$ 及 $118.15 \%$, 由此认为C部分可 能为非必需的结构. 我们继续简化结构, 设计了 linker 部分苯环上没有或仅有常见取代基的目标分子 TM4 系列(图3,37个分子).

鉴于pioglitazone含有吡啶环的事实, 进一步将目 标化合物 TM4系列的左端取代苯环变换为芳杂环, 设 计了目标分子TM5系列(图4,9个分子).

我们共设计合成了 62 个分子, 发现 18 个分子的 PPAR相对激动活性超过 $100 \%$, 为后续研究提供了新 的思路.

\section{2 实验部分}

\section{1 检测仪器和主要试剂}

精密显微熔点测定仪 (X-6, 北京福凯仪器有限公 司); 傅里叶变换红外光谱仪(GX, Perkin Elmer, 美国); 超导核磁共振波谱仪(AV-300, Bruker, 德国); 高分辨 质谱仪(HRFTIC-MS Varian 7.0, Varian, 美国); 除3-氟 苯甲醛(Fluka, 分析纯, 美国)、3-甲基苯甲醛(Merck, 分析纯, 德国)、2-噻吩甲醛(Alfa Acear, 分析纯, 英国) 外, 其他原料均为市售化学纯产品, 溶剂均为市售分 析纯产品.

\section{2 化合物的合成}

TM1 TM3 系列目标化合物的关键合成步骤是 取代芳香醛与噻唑烷-2,4-二酩(IM1)的活泼亚甲基发 生Knoevenagel 反应得到 5-芳亚甲基噻唑烷-2,4-二酮 (IM2 IM5). 按其结构, 可以有两条合成路线, 不同合 成路线的差别在于酚羟基的烷基化顺序: 含羟基的醛 (酚醛)与IM1 缩合后再进行酚羟基的烷基化(路线2), 存在酚羟基与噻唑烷环中NH双烷基化的可能; 酚醛 烷基化后再与IM1缩合(路线1), 理论上产物更为单一, 有利于高纯度、高收率地合成目标化合物, 故采用路 线 1 (图5).

目标化合物 TM4 与 TM5系列的合成采用芳香醛、 杂环醛与中间体IM1 的活泼亚甲基直接发生Knoevenagel反应得到.

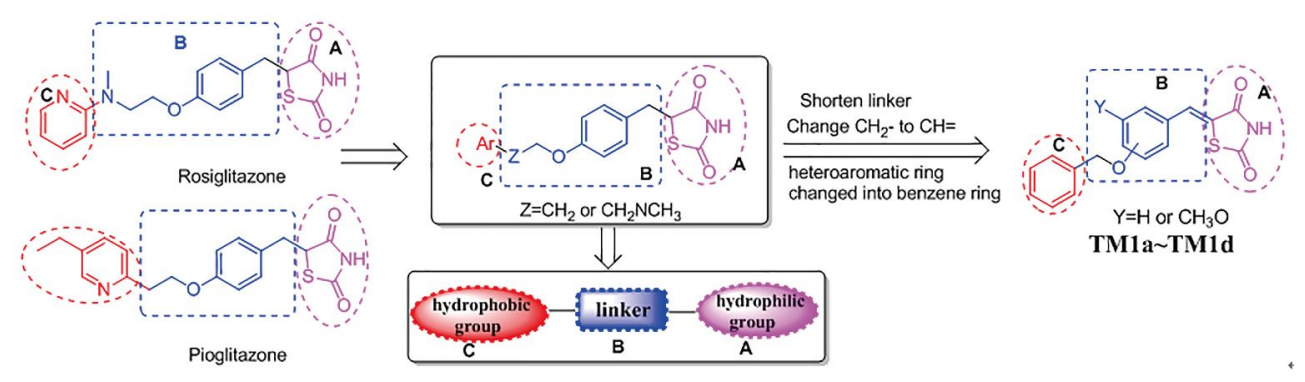

图 1 目标化合物 TM1系列的设计(网络版彩图)

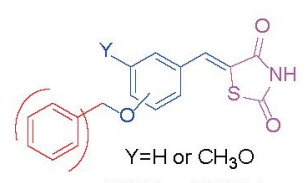

TM1a TM1d

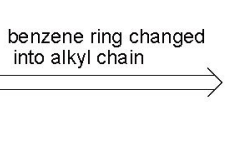

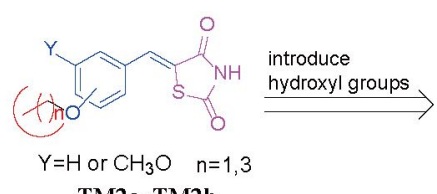

TM2a TM2h

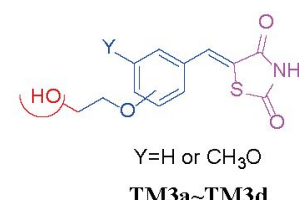

TM3a TM3d

图 2 目标化合物 TM2 和 TM3 系列的设计(网络版彩图) 


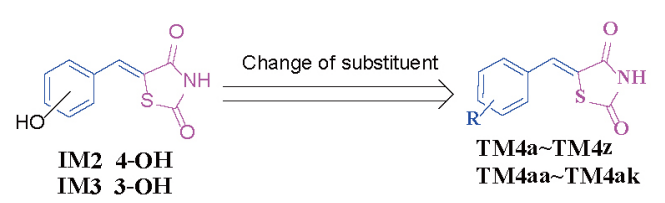

图 3 目标化合物 TM4系列的设计(网络版彩图)

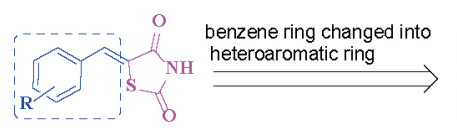

TM4a TM4z

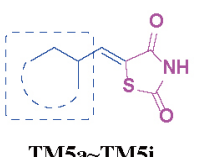

TM5a TM5i

图 4 目标化合物TM5系列的设计(网络版彩图)

固相合成法: 于研钭中加入醛 $11 \mathrm{mmol}$ 、中间体 IM1 $10 \mathrm{mmol}$ 和无水 $\mathrm{NaOAc} 10 \mathrm{mmol}$, 混合研磨至粉末 状后, 转入 $100 \mathrm{~mL}$ 圆底烧瓶, 油浴 $125^{\circ} \mathrm{C}$ 反应, 完全固 化时停止反应, 趁热加入二甲基甲酰胺(DMF)使固体 完全溶解, 加入 $50 \mathrm{~mL} \mathrm{H}_{2} \mathrm{O}$ 析出大量固体, $2 \mathrm{NHCl}$ 溶液 调节 $\mathrm{pH} 5 \sim 6$, 室温摚拌 $30 \mathrm{~min}, 4^{\circ} \mathrm{C}$ 静置, 抽滤, 滤饼水 洗, $100^{\circ} \mathrm{C}$ 干燥, $\mathrm{Et}_{2} \mathrm{O} / \mathrm{EA}=2: 1(v / v)$ 混合溶剂 $6 \sim 8 \mathrm{~mL}$ 分 散过夜, 抽滤, 干燥, 即得目标化合物.

液相合成法: 于 $100 \mathrm{~mL}$ 圆底瓶中加入醛 $1 \mathrm{mmol}$ 、 $\mathrm{CH}_{3} \mathrm{CH}_{2} \mathrm{OH} 5 \mathrm{~mL}$, 室温摚拌下加入哌啶 $1.2 \mathrm{mmol}$ 和 $1 \mathrm{mmol}$ 中间体 IM1, 加热至沸腾使大部分中间体 IM1 溶解, $80^{\circ} \mathrm{C}$ 回流反应, 薄层色谱(TLC)监测反应进程至 反应完全, 冷却, 过滤, 滤饼依次用 $\mathrm{H}_{2} \mathrm{O} 、 \mathrm{CH}_{3} \mathrm{OH}$ 洗涤 2次, 干燥, $\mathrm{DMF} / \mathrm{H}_{2} \mathrm{O}$ 重结晶, 即得目标化合物.

中间体 IM1 IM21 合成步骤与目标化合物 TM1 TM5系列的结构表征见网络版补充材料.

\subsection{PPAR激动活性测定}

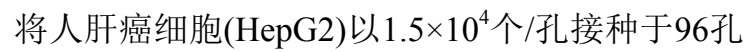
板后, 用含 $100 \mathrm{U} / \mathrm{mL}$ 链霉素和青需素的低糖杜尔伯科
极限必需培养基(DMEM), 在温度 $37^{\circ} \mathrm{C} 、 \mathrm{CO}_{2}$ 气体体积 分数为 $5 \%$ 的条件下培养过夜, 参照转染试剂说明书进 行质粒转染, 转染的质粒包括带有PPRE和萤火虫荧光 素酶(Luc)报告基因的质粒pPPRE-Luc以及用作转染内 参照的带有海肾荧光素酶报告基因的质粒phRL-TK. 转染 $24 \mathrm{~h}$ 后换用含有 $10 \mu \mathrm{g} / \mathrm{mL}$ 待测样品的培养基, 同 时设立空白对照(未转染的细胞)、阴性对照(转染的 细胞加入不含样品的培养基)和阳性对照(转染的细 胞加入含有 $0.78 \mu \mathrm{g} / \mathrm{mL}$ 吡格列酮的培养基), 继续培养 $24 \mathrm{~h}$ 后用双苂光素酶报告基因检测试剂盒(Promega, 美 国)检测苂光素酶活性, 根据检测到的化学发光强度 $L$

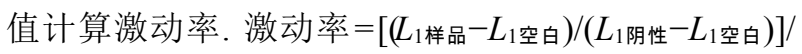
$\left[\left(L_{2 \text { 样品 }}-L_{2}\right.\right.$ 空白 $) /\left(L_{2}\right.$ 阴性 $-L_{2}$ 空白 $\left.)\right] \times 100 \%$, 其中 $L_{1}$ 为萤火虫荧 光素酶的化学发光强度, $L_{2}$ 为内参照海肾苂光素酶的 化学发光强度, 结果以样品与阳性对照的相对激动率 表示. 每个样品设双复孔, 重复两次.

\section{4 半数有效浓度的测定}

取部分PPRE相对激动率大于 $70 \%$ 的样品, 每个 样品梯度稀释成 6 个浓度, 每个浓度设双复孔, 测定 PPAR激动率, 再应用Xlfit软件中的4 Parameter Logistic Model计算半数有效浓度 $\left(\mathrm{EC}_{50}\right)$.

\section{3 结果与讨论}

\section{1 化合物的合成结果与讨论}

本实验共合成62个化合物, 收率为 $26.5 \%$ 90.0\%。 所得新化合物结构经核磁共振氢谱 ( ${ }^{1} \mathrm{H}$ NMR)、核磁共 振碳谱 $\left({ }^{13} \mathrm{C} N M R\right)$ 和高分辨质谱(HR-MS)证实, 已知化 合物经熔点测定、红外光谱(IR)、 ${ }^{1} \mathrm{H} N M R$ 或 ${ }^{13} \mathrm{C} N M R$ 表征确定. 中间体IM1 IM21与目标化合物 TM1 TM5 系列的具体合成结果与讨论见网络版补充材料.

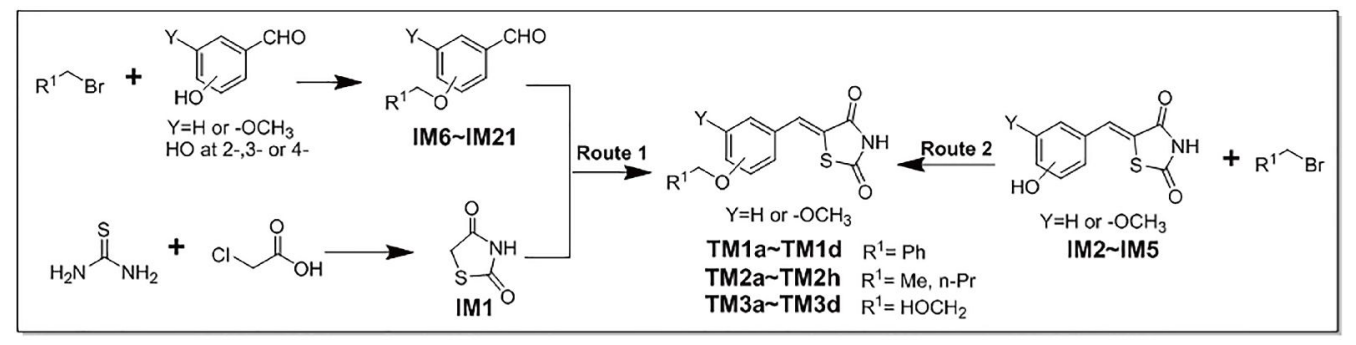

图 5 目标化合物 TM1 TM3 系列合成路线

1116 


\section{2 生物活性分析}

过氧化物酶体增殖物激活受体(PPAR) 是细胞核 内受体, 分为 $\alpha 、 \beta / \delta$ 和 $\gamma 3$ 种亚型, 与配体结合后被激 活, 可与维甲类X受体 (RXR) 结合形成异二聚体, 再 与靶基因启动子区域的PPRE结合, 调控靶基因的转

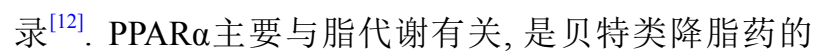
作用靶点; PPAR $\delta$ 分布广泛, 作用尚不清楚; PPAR $\gamma$ 具 有多种生物学效应, 在糖类代谢、脂肪合成、脂肪细
胞分化、动脉粥样硬化、炎性反应和免疫中起重要 作用, 已成为肥胖症、糖尿病、炎症、肿瘤等疾病治 疗药物篎选的重要靶标. PPRE是评价潜在PPAR激动 剂的有效工具. 本文通过检测PPRE激动活性来衡量 化合物的抗糖尿病活性.

以吡格列酮 $(0.78 \mu \mathrm{g} / \mathrm{mL}, 100 \%)$ 为阳性参照物, 测 定样品 $(10 \mu \mathrm{g} / \mathrm{mL})$ 的PPRE激动活性(表1 3). 篮选结果 显示, 62 个目标化合物中有 21 个化合物的PPRE相对激 动活性超过了 $70 \%$, 其中 18 个化合物的相对激动活性

表 1 目标化合物 TM1 TM3 系列的PPRE相对激动活性结果

\begin{tabular}{ccc||ccc}
\hline TM & $C \log P$ & Relative activation $(\%)$ & TM & $C \log P$ & Relative activation $(\%)$ \\
\hline $\mathbf{1 a}$ & 3.4 & -33.73 & $\mathbf{2 e}$ & 3.0 & 14.95 \\
$\mathbf{1 b}$ & 3.4 & -1.61 & $\mathbf{2 f}$ & 3.0 & 60.58 \\
$\mathbf{1 c}$ & 3.4 & 51.22 & $\mathbf{2 g}$ & 3.0 & 68.62 \\
$\mathbf{1 d}$ & 3.2 & 64.99 & $\mathbf{2 h}$ & 2.8 & -22.01 \\
$\mathbf{2 a}$ & 2.0 & -1.80 & $\mathbf{3 a}$ & 1.0 & 17.29 \\
$\mathbf{2 b}$ & 2.0 & 62.42 & $\mathbf{3 b}$ & 1.0 & 23.05 \\
$\mathbf{2 c}$ & 2.0 & 125.30 & $\mathbf{3 c}$ & 1.0 & 100.53 \\
$\mathbf{2 d}$ & 1.9 & 55.86 & $\mathbf{3 d}$ & 0.95 & -24.53 \\
pioglitazone & 3.3 & 100 & - & - & - \\
\hline
\end{tabular}

表 2 目标化合物 TM4 系列的PPRE相对激动活性结果

\begin{tabular}{|c|c|c|c|c|c|c|c|}
\hline $\mathrm{TM}$ & $\mathrm{R}$ & $C \log P$ & PPRE (\%) & $\mathrm{TM}$ & $\mathrm{R}$ & $C \log P$ & PPRE (\%) \\
\hline $4 a$ & $4-\mathrm{NO}_{2}$ & 1.8 & 162.67 & $4 t$ & 2,4-diCl & 3.1 & 43.69 \\
\hline $4 b$ & $3-\mathrm{NO}_{2}$ & 1.8 & 57.86 & $4 u$ & 2,3-diCl & 3.1 & 52.19 \\
\hline $4 c$ & $2-\mathrm{NO}_{2}$ & 1.8 & 142.69 & $4 v$ & 2,6-diCl & 3.1 & 18.10 \\
\hline $4 d$ & $4-\mathrm{CN}$ & 1.7 & 166.87 & $4 w$ & 3,4-diCl & 3.1 & 59.40 \\
\hline $4 e$ & $3-\mathrm{CN}$ & 1.7 & 58.70 & $4 x$ & $\mathrm{H}$ & 1.8 & 125.26 \\
\hline $4 f$ & $4-\mathrm{F}$ & 2.0 & 182.25 & $4 y$ & $4-\mathrm{CH}_{3}$ & 2.4 & 204.70 \\
\hline $4 \mathrm{~g}$ & $3-\mathrm{F}$ & 2.0 & 46.35 & $4 z$ & $3-\mathrm{CH}_{3}$ & 2.4 & 62.30 \\
\hline $4 \mathrm{~h}$ & $2-\mathrm{F}$ & 2.0 & 173.97 & 4aa* & $4-\mathrm{OH}$ & 1.5 & 105.76 \\
\hline $4 \mathbf{i}$ & $4-\mathrm{CF}_{3}$ & 2.7 & 239.77 & $4 a b^{*}$ & $3-\mathrm{OH}$ & 1.5 & 118.15 \\
\hline $4 j$ & $3-\mathrm{CF}_{3}$ & 2.7 & 15.75 & $4 a^{*}$ & $2-\mathrm{OH}$ & 1.5 & 51.36 \\
\hline $4 k$ & $2-\mathrm{CF}_{3}$ & 2.7 & 54.71 & 4ad & 4- $\mathrm{CH}_{3} \mathrm{O}$ & 1.7 & 121.39 \\
\hline 41 & $4-\mathrm{Br}$ & 2.6 & 88.49 & $4 \mathrm{ae}$ & $3-\mathrm{CH}_{3} \mathrm{O}$ & 1.7 & 124.35 \\
\hline $4 m$ & $3-\mathrm{Br}$ & 2.6 & 127.06 & $4 \mathrm{af}$ & $2-\mathrm{CH}_{3} \mathrm{O}$ & 1.7 & 58.97 \\
\hline $4 n$ & $2-\mathrm{Br}$ & 2.6 & 9.80 & 4ag & 2,4- $\mathrm{diCH}_{3} \mathrm{O}$ & 1.5 & 51.00 \\
\hline 40 & $3-\mathrm{Br}-4-\mathrm{F}$ & 2.8 & 50.63 & $4 \mathrm{ah}$ & $2,5-\mathrm{diCH}_{3} \mathrm{O}$ & 1.5 & 65.44 \\
\hline $4 p$ & 2-Cl-4-F & 2.6 & 167.98 & 4ai & $3,4-\mathrm{diCH}_{3} \mathrm{O}$ & 1.5 & 44.93 \\
\hline $4 q$ & $4-\mathrm{Cl}$ & 2.5 & 100.68 & 4aj & 3,4,5-triCH${ }_{3} \mathrm{O}$ & 1.4 & -5.24 \\
\hline $4 r$ & $3-\mathrm{Cl}$ & 2.5 & 125.03 & $4 a^{a)}$ & $3-\mathrm{CH}_{3} \mathrm{O}-4-\mathrm{OH}$ & 1.4 & 0.82 \\
\hline $4 s$ & $2-\mathrm{Cl}$ & 2.5 & 97.83 & pioglitazone & - & 3.3 & 100 \\
\hline
\end{tabular}

a)为了便于构效分析, 将中间体IM2 IM5的4个分子合并到TM4系列(分别对应于TM4aa、TM4ab、TM4ac、TM4ak) 
黄敏等: 具有激动过氧化物酶体增殖物激活受体活性的 5-芳(杂环)亚甲基噻唑烷-2,4-二酮类化合物的研究

表 3 目标化合物 TM5系列的PPRE相对激动活性结果

\begin{tabular}{ccc||ccc}
\hline $\mathrm{TM}$ & $C \log P$ & Relative activation (\%) & $\mathrm{TM}$ & $C \log P$ & Relative activation $(\%)$ \\
\hline $\mathbf{5 a}$ & 1.8 & 7.34 & $\mathbf{5 f}$ & 0.6 & 28.24 \\
$\mathbf{5 b}$ & 0.9 & -30.29 & $\mathbf{5 g}$ & 0.6 & 35.58 \\
$\mathbf{5 c}$ & 1.9 & 54.13 & $\mathbf{5 h}$ & 0.9 & 46.15 \\
$\mathbf{5 d}$ & 3.8 & 6.54 & $\mathbf{5 i}$ & 1.5 & 65.06 \\
$\mathbf{5 e}$ & 2.7 & 47.61 & pioglitazone & 3.3 & 100 \\
\hline
\end{tabular}

超过 $100 \%$, 化合物 TM4i和 TM4y 的相对激动活性分别 达到了 $239.77 \%$ 和 $204.70 \%$.

\subsection{1 化合物 TM1 TM3 系列的PPRE激动活性}

初步活性数据显示, TM1 系列化合物PPRE激动活 性较差, 最高者TM1d仅 $64.99 \%$, 小于更低浓度下的对 照药物吡格列酮. 计算 TM1 系列与吡格列酮的 $C \log P$ (利用美国礼来制药公司提供的在线软件Plexus), 发现 $C \log P$ 值差别很小, 因此 $C \log P$ 不是影响 TM1系列活性 的主要因素. TM2 系列化合物的活性数据显示, 当取 代基为乙基且处于4位时(TM2c)活性达到125.3\%。通 过SimBioSys 公司的eHiTS软件, 对 TM2c进行了蛋白 质(PPAR $\gamma$, PDB code $2 \mathrm{PRG}^{[13]}$ ) 对接, 利用Pymol软件绘 图(图6). 从图6可看出, TM2c在蛋白空腔的位置与吡 格列酮相似、且氢键的作用位点相同, 但 TM2c的疏 水端体积较小, 可能是 TM2c 的活性比吡格列酮好的 原因。

在 TM2系列的基础上引入羟基以增加亲水性(降 低 $C \log P$ ) 得到 $\mathbf{T M} 3$ 系列化合物. 活性数据显示, 降低 $C \log P$ 并未达到增加活性的目的. 图 6显示, 化合物 TM3c 与蛋白空腔的结合区域发生了改变(结合位点 在蛋白U型腔侧面), 且与水分子形成了弱的氢键作用, 这些可能是TM3c活性低于 TM2c的原因.

构效关系分析发现, TM1 TM3 系列的活性大小 关系均为 2-<3-<4-, 表明苯环上取代基处于4-位时, 具 有更好的PPRE激动活性, 这与文献[14 17]规律一致.

\subsection{2 化合物TM4系列的PPRE激动活性}

在样品浓度为 $10 \mu \mathrm{g} / \mathrm{mL}$ 下, TM4系列分子整体活 性均较好, 其中 16 个化合物的PPRE相对激动活性超过 了 $100 \%$, 以 TM4i $\left(4-\mathrm{CF}_{3}\right) 、 \mathbf{T M} 4 \mathbf{y}\left(4-\mathrm{CH}_{3}\right)$ 的活性最高, 分别达到了 $239.77 \%$ 和 $204.70 \%$, 这足以说明5-芳亚甲 基噻唑烷-2,4-二酮片段具有明显的抗糖尿病活性, 可
作为抗糖尿病先导分子进一步研究.

构效关系分析发现, 含有强吸电子基 $\mathrm{NO}_{2}$ 和给电 子基 $\mathrm{CH}_{3} \mathrm{O}$ 的化合物其活性均能达到较高值, 如 TM4a $\left(4-\mathrm{NO}_{2}\right)$ 和 TM4b $\left(2-\mathrm{NO}_{2}\right) 、$ TM4ad $\left(4-\mathrm{CH}_{3} \mathrm{O}\right)$ 和 TM4ae $\left(3-\mathrm{CH}_{3} \mathrm{O}\right)$ 的活性分别达到了 $162.67 \%$ 和 $142.69 \%$ 、 121.39\%和 $124.35 \%$; 不含取代基的化合物 $\mathbf{T M 4 x}(\mathrm{R}=\mathrm{H})$ 其活性为 $125.26 \%$, 活性大于 $125.26 \%$ 的化合物一般均 含有吸电子取代基, 而活性介于 $125.26 \%$ 100\%的化 合物多数含有给电子取代基或氯原子, 从此角度分 析, 吸电子取代基化合物的活性比给电子取代基化 合物的活性稍强.

研究取代基位置对活性的影响发现, 含强吸电子 基 $\mathrm{NO}_{2} 、 \mathrm{CN} 、 \mathrm{~F} 、 \mathrm{CF}_{3}$ 和弱供电子基 $\mathrm{CH}_{3}$ 的化合物, 其 PPRE活性变化规律为4->2->3-, 活性最好的两个化合 物, TM4i和TM4y均为4位取代的化合物; 对于含 $\mathrm{Br}$ 、 $\mathrm{Cl} 、 \mathrm{CH}_{3} \mathrm{O}$ 和 $\mathrm{OH}$ 取代基的化合物, 其活性强弱顺序是 3->4->2-, 表明取代基位置是影响活性的关键因素, 且取 代基的种类对活性也有一定的影响. 另外, 含有两个或 3 个取代基的化合物(TM4t TM4w, TM4ag TM4ak), 其活性明显低于只含一个取代基的化合物, 表明空间 体积亦是影响活性变化的重要因素.

上述研究表明, 芳环上取代基的位置、种类、电 性以及分子空间体积均是影响化合物PPRE激动活性 的因素. 这可能与目标分子同PPAR靶点结合性能相关. 对接图7表明, 大部分高活性化合物与蛋白的结合位 点均在蛋白的U型腔底部, 而取代基处于2-位(TM4c) 或无取代基(TM4x)时, 化合物与蛋白的结合位点在蛋 白的U型腔侧面.

\subsection{3 化合物TM5系列的PPRE激动活性}

所有杂环醛生成的目标分子TM5系列, 其PPRE 活性均较差(最高者仅为 $65.06 \%$ ). 可能和上市药物结 构中与TZD环的连接处为柔性的 $\mathrm{CH}_{2}$ 、目标分子 TM5 
系列在此处的连接为刚性的 $\mathrm{CH}=$ 有关.

\subsection{4 部分高活性化合物的 $\mathrm{EC}_{50}$ 值测定结果}

表4为部分高活性化合物的 $\mathrm{EC}_{50}$ 值的测定结果. 表4表明, 大部分高活性目标化合物的最大激动率高 于吡格列酮, 但高活性目标化合物的 $\mathrm{EC}_{50}$ 值却大于吡 格列酮, 因而有待进一步改构以提高活性. 部分分子
的体内测试还在进行中.

\section{3 毒性预测}

糖尿病是慢性疾病, 需要长期服药, 因而糖尿病 类药物必须无毒或低毒性. 为了预知相对PPRE激动 活性超过 $100 \%$ 的 18 个化合物的安全性,我们使用美国 Simulations Plus公司的毒性预测软件ADMET Predictor 8.0 预测了这些化合物的毒性(图8).

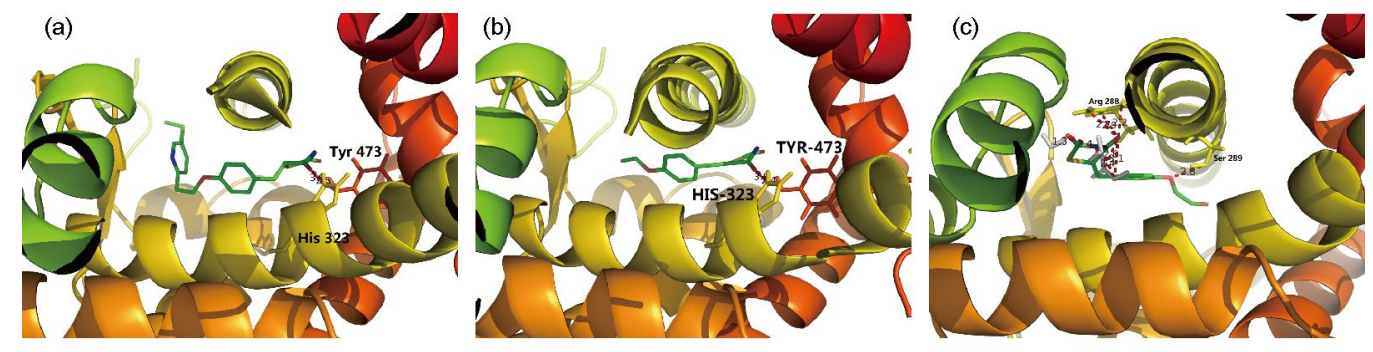

图 6 吡格列酮(a)、TM2c (b)和TM3c (c) 与PPAR蛋白的对接图(网络版彩图)
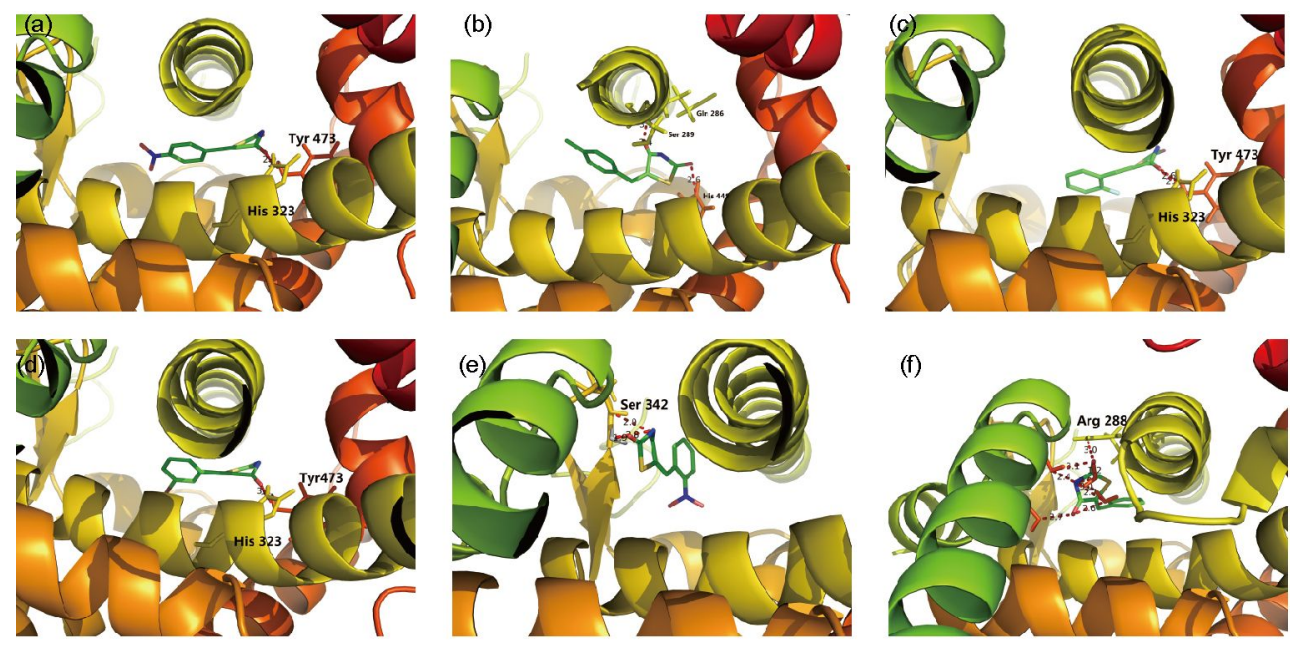

图 7 化合物TM4a (a), TM4y (b), TM4h (c), TM4m (d), TM4c (e), TM4x (f) 与蛋白对接图(网络版彩图)

表 4 部分高活性化合物的 $\mathrm{EC}_{50}$ 值的测定结果

\begin{tabular}{cccc||cccc}
\hline $\mathrm{TM}$ & Max conc. & $\mathrm{EC}_{50}(\mu \mathrm{g} / \mathrm{mL})$ & Max activation $(\%)$ & $\mathrm{TM}$ & Max conc. & $\mathrm{EC}_{50}(\mu \mathrm{g} / \mathrm{mL})$ & Max activation $(\%)$ \\
\hline $\mathbf{2 c}$ & 10.00 & 1.91 & 185.81 & $\mathbf{4 p}$ & 10.00 & 8.35 & 141.38 \\
$\mathbf{3 c}$ & 10.00 & 1.12 & 186.51 & $\mathbf{4 x}$ & 10.00 & 3.18 & 157.02 \\
$\mathbf{4 a}$ & 10.00 & 6.12 & 122.22 & $\mathbf{4 y}$ & 10.00 & 2.02 & 201.54 \\
$\mathbf{4 c}$ & 10.00 & 1.17 & 132.31 & $\mathbf{4 a b}$ & 10.00 & 4.42 & 93.85 \\
$\mathbf{4 d}$ & 10.00 & 2.17 & 171.51 & $\mathbf{4 a d}$ & 10.00 & 2.00 & 160.83 \\
$\mathbf{4 f}$ & 10.00 & 4.11 & 88.57 & $\mathbf{4 a e}$ & 10.00 & 3.30 & 112.50 \\
$\mathbf{4 h}$ & 10.00 & 3.32 & 102.20 & pioglitazone & 0.78 & 0.087 & 123.88 \\
$\mathbf{4 i}$ & 10.00 & 1.42 & 264.26 & - & - & - & - \\
\hline
\end{tabular}




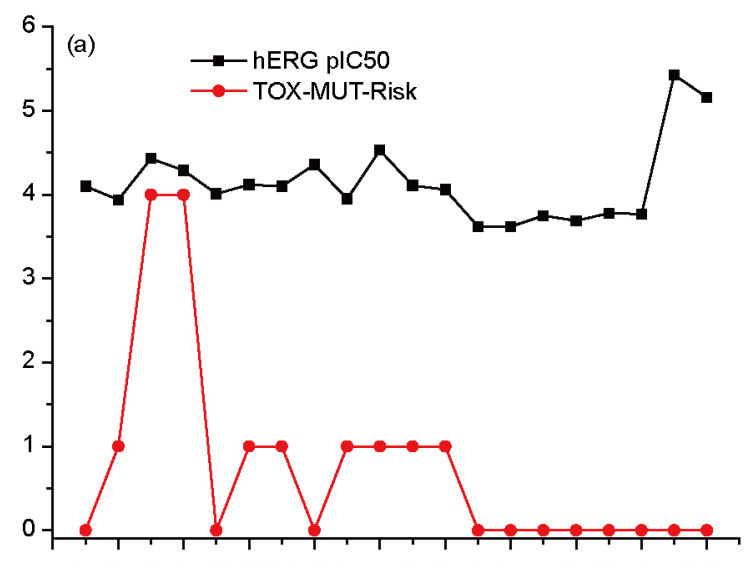

2c 3c 4a 4c 4d 4f 4h 4i 4m 4p 4q 4r 4x 4y4aa4ab4ad4aeR $P$
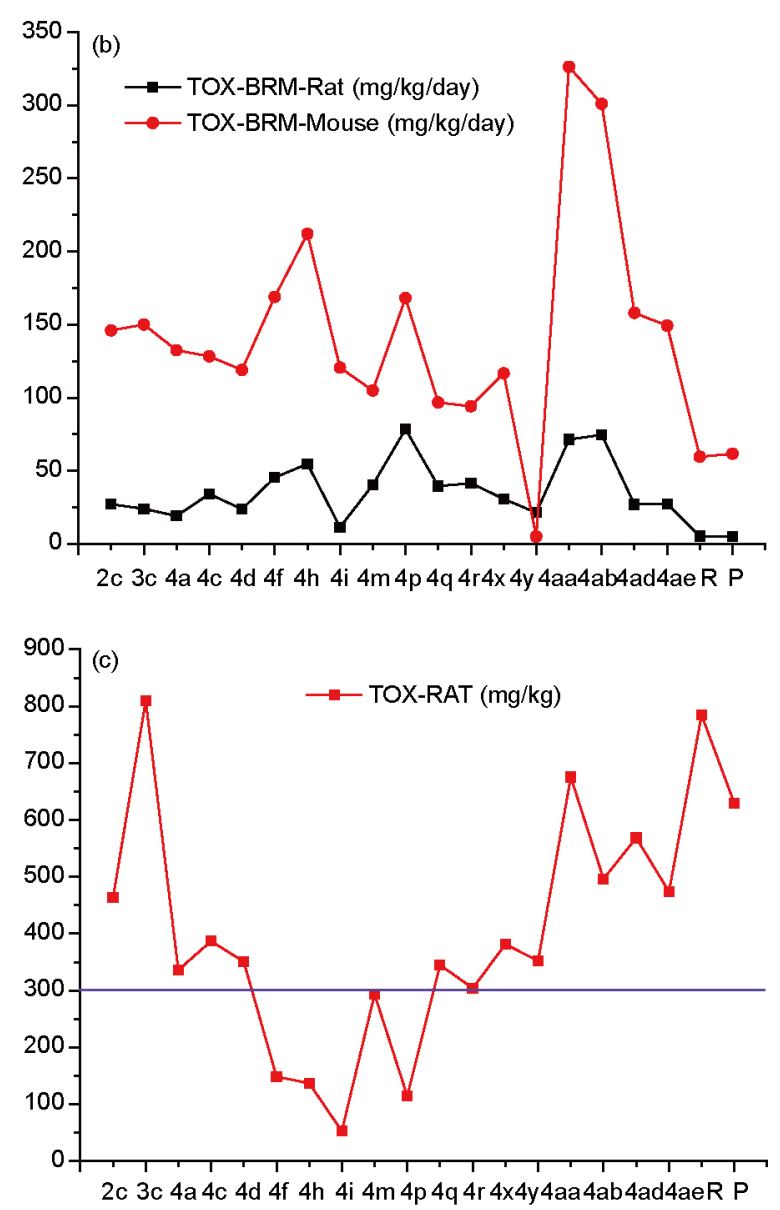

图 8 (a) 高活性化合物的心脏毒性与致突变性预测结果图; (b) 高活性化合物在大鼠和小鼠模型的致癌性预测结果图;

(c) 高活性化合物的大鼠急性毒性预测结果图(P表示吡格列 酮, R表示罗格列酮) (网络版彩图)

糖尿病治疗是否伴随心血管类疾病, 一直备受关 注. 可喜的是, 目前发现了不仅没有心血管疾病风险
而且还对心血管疾病有益的药物, SGLT-2抑制剂恩格 列净、GLP-1受体激动剂利拉鲁肽均为对心血管有益 的糖尿病药物. hERG定量预测化合物的心脏毒性, 规 定 $\mathrm{pIC}_{50} \leq 6 \mathrm{~mol} / \mathrm{L}$ 为正常值. 图8显示, 所得高活性分子 的 $\mathrm{pIC}_{50}<6 \mathrm{~mol} / \mathrm{L}$, 且都小于上市药物罗格列酮与吡格 列酮, 可能没有心脏毒性. TM4系列高活性化合物中, 苯环上取代基为吸电子基的化合物, 其 $\mathrm{hERG}$ 心脏毒 性要大于取代基为供电子基的化合物.

TOX-MUT-Risk预测化合物在不同沙门菌属试验 中的致突变可能性, 成药性要求 TOX-MUT-Risk $\leq 2$. 从图 8 发现, 高活性化合物中只有 TM4a $\left(4-\mathrm{NO}_{2}\right)$ 与 TM4c $\left(2-\mathrm{NO}_{2}\right)$ 的致突变性系数大于 2 , 表明绝大多数 分子的致突变可能性较低.

近年的研究证实, 降糖药物二甲双胍不仅能降低 糖尿病患者心梗死的风险, 而且能抑制肿瘤生长 ${ }^{[18]}$. TOX-BRM-Rat和TOX-BRM-Mouse可预测化合物的致 癌性, 成药性要求药物分子半数致癌浓度 $\left(\mathrm{TD}_{50}\right)$ 的预测 值应该 $\geq 4 \mathrm{mg} / \mathrm{kg} / \mathrm{d}$ (大鼠动物模型)或 $\geq 25 \mathrm{mg} / \mathrm{kg} / \mathrm{d}$ (小鼠 动物模型). 预测高活性化合物在大鼠(TOX-BRM-Rat) 和小鼠(TOX-BRM-Mouse)模型上的致癌性, 发现除 TM4y 以外, 所有分子均满足要求且 $\mathrm{TD}_{50}$ 值大于两种 上市药物.

TOX-RAT预测化合物的急性毒性, 以半数致死 浓度 $\mathrm{LC}_{50}$ (动物模型大鼠) 表示, 要求大多数药物分子 的 $\mathrm{LC}_{50}$ 预测值应该 $\geq 300 \mathrm{mg} / \mathrm{kg}$. 从图 8 发现, TM4f、

TM4h、TM4i、TM4m和TM4p的 $\mathrm{LC}_{50}<300 \mathrm{mg} / \mathrm{kg}$, 可 能有急性毒性, 而其他分子的急性毒性可能较小.

总体来看, 相对PPRE激动活性超过 $100 \%$ 的 18 个 化合物中, 大多数分子的毒性较低, 且小于两个上市 药物, 有进一步开发的前景.

\section{4 结论}

为系统研究TZD类分子的PPRE激动活性, 本文 设计合成了 62 个 5 -芳(杂环)亚甲基噻唑烷-2,4-二酮 类化合物. 体外PPRE激动活性结果显示, 在 $10 \mu \mathrm{g} / \mathrm{mL}$ 检测浓度下, 18 个化合物的PPRE相对激动活性超过 $100 \%$, 其中化合物 TM4i 和 TM4y 分别达到了 $239.77 \%$ 和 $204.70 \%$, 远超过强效的阳性对照物吡格列酮. 分子 毒性预测显示, 大多数高活性分子的毒性较低. 本研 究为研发高效低毒、合成简易、成本低廉的抗糖尿 病药物提供了新的研究思路. 
致谢感谢西南大学化学化工学院王宁老师测试核磁数据、元为老师测试红外数据; 感谢成都地奥制药集团有限 公司晏菊芳博士等测试PPRE活性数据.

\section{补充材料}

本文的补充材料见网络版 chemcn.scichina.com. 补充材料为作者提供的原始数据, 作者对其学术质量和内容负责.

\section{参考文献}

中华医学会糖尿病学分会. 中国2型糖尿病防治指南(2013年). 北京大学医学出版社, 2014. 14

2 Quianzon CCL, Cheikh IE. J Commun Hosp Int Med Perspect, 2012, 2: 19081

3 Watkins PB, Whitcomb RW. N Engl J Med, 1998, 338: 916-917

4 Balfour JAB, Plosker GL. Drugs, 1999, 57: 921-930

5 Yasmin S, Jayaprakash V. Eur J Med Chem, 2017, 126: 879-893

6 Psaty BM, Furberg CD. N Engl J Med, 2007, 356: 2522-2524

7 刘红萍. 新型抗糖尿病药物的设计、合成和生物活性的初步研究. 硕士学位论文. 重庆: 西南大学, 2008. 111-121

8 许菍. 基于抗糖尿病活性化合物的设计、合成和生物活性初步研究. 硕士学位论文. 重庆: 西南大学, 2009. 81-113

9 Xia Z, Knaak C, Ma J, Beharry ZM, McInnes C, Wang W, Kraft AS, Smith CD. J Med Chem, 2009, 52: 74-86

10 Heng S, Tieu W, Hautmann S, Kuan K, Pedersen DS, Pietsch M, Gütschow M, Abell AD. Bioorg Med Chem, 2011, 19: 7453-7463

11 Żesławska E, Nitek W, Tejchman W. J Chem Crystallogr, 2015, 45: 151-157

12 Gross B, Staels B. Best Prac Res Clin Endoc Metab, 2007, 21: 687-710

13 Nolte RT, Wisely GB, Westin S, Cobb JE, Lambert MH, Kurokawa R, Rosenfeld MG, Willson TM, Glass CK, Milburn MV. Nature, 1998, 395: $137-143$

14 杨艳, 晏菊芳, 范莉, 陈欣, 蒋理, 杨大成. 药学学报, 2012, 47: 1630-1639

15 周礼江, 晏菊芳, 张坤, 范莉, 陈欣, 杨大成. 药学学报, 2013, 48: 1570-1578

16 张坤, 晏菊芳, 唐雪梅, 刘红萍, 范莉, 周光明, 杨大成. 药学学报, 2011, 46: 412-421

17 Wang H, Yan J, Song X, Fan L, Xu J, Zhou G, Jiang L, Yang D. Bioorg Med Chem, 2012, 20: 2119-2130

18 Li D, Yeung SCJ, Hassan MM, Konopleva M, Abbruzzese JL. Gastroenterology, 2009, 137: 482-488 


\title{
Design, synthesis and PPAR activities of 5-arylmethylene and 5-heteroaromatic methylene thiazolidine-2,4-diones
}

\author{
Min Huang ${ }^{\dagger}$, Kun Zhang ${ }^{\dagger}$, Li Fan, Lijiang Zhou, Dacheng Yang* \\ Institute of Bioorganic and Medicinal Chemistry, Key Laboratory of Applied Chemistry of Chongqing Municipality, School of Chemistry and \\ Chemical Engineering, Southwest University, Chongqing 400715, China \\ $\dagger$ These authors contributed equally to this work \\ *Corresponding author (email: hxydc@swu.edu.cn)
}

\begin{abstract}
A series of antidiabetic drug molecule TM1 TM3 were designed via considering thiazolidine-2,4-dione as the basic structural unit. Peroxisome proliferator-activated receptor response element (PPRE) relative agonist activity in vitro showed that intermediate IM2 and IM3, target molecules TM2c and TM3c with agonist activities of 105.76\%, $118.15 \%, 125.3 \%$ and $100.53 \%$, respectively (reference drug is pioglitazone, $0.78 \mu \mathrm{g} / \mathrm{mL}$ ). Furthermore, a series of TM4 and TM5 were also synthesized, and the PPRE relative agonist activities of the 16 molecules of TM4 was higher than 100\%. Among them, TM4i and TM4y had the highest PPRE activity with $239.77 \%$ and 204.70\%, respectively. And the toxicity of high active molecules were weaker than that of pioglitazone. Through this study, we found potential PPAR lead molecules, which provided new ideas for the development of simpler diabetes drugs.
\end{abstract}

Keywords: diabetes mellitus, peroxisome proliferator-activated receptor, thiazolidine-2,4-dione, toxicity prediction, synthesis

doi: $10.1360 / \mathrm{N} 032017-00070$ 